\title{
Collisional damping rates for plasma waves
}

S. F. Tigik' , L. F. Ziebell', and P. H. Yoon'

Citation: Physics of Plasmas 23, 064504 (2016); doi: 10.1063/1.4953802

View online: http://dx.doi.org/10.1063/1.4953802

View Table of Contents: http://aip.scitation.org/toc/php/23/6

Published by the American Institute of Physics

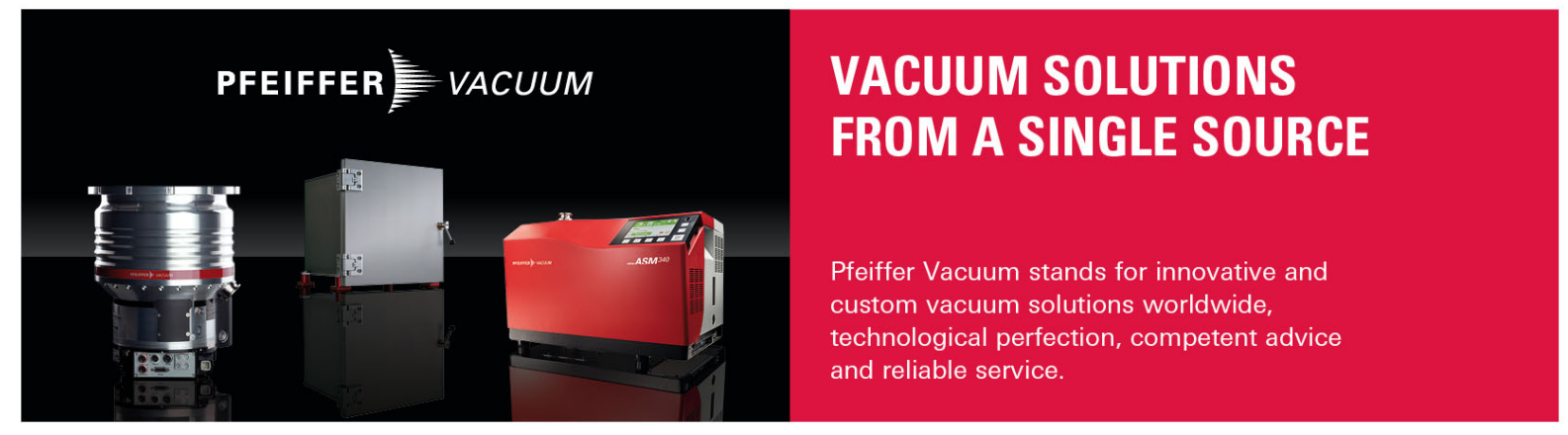




\title{
Collisional damping rates for plasma waves
}

\author{
S. F. Tigik, ${ }^{1, a)}$ L. F. Ziebell, ${ }^{1, b)}$ and P. H. Yoon ${ }^{2,3, c)}$ \\ ${ }^{1}$ Instituto de Física, Universidade Federal do Rio Grande do Sul, 91501-970 Porto Alegre, Rio Grande do Sul, \\ Brazil \\ ${ }^{2}$ Institute for Physical Science and Technology, University of Maryland, College Park, Maryland 20742, \\ USA \\ ${ }^{3}$ School of Space Research, Kyung Hee University, Yongin, Gyeonggi 446-701, South Korea
}

(Received 22 May 2016; accepted 31 May 2016; published online 14 June 2016)

The distinction between the plasma dynamics dominated by collisional transport versus collective processes has never been rigorously addressed until recently. A recent paper [P. H. Yoon et al., Phys. Rev. E 93, 033203 (2016)] formulates for the first time, a unified kinetic theory in which collective processes and collisional dynamics are systematically incorporated from first principles. One of the outcomes of such a formalism is the rigorous derivation of collisional damping rates for Langmuir and ion-acoustic waves, which can be contrasted to the heuristic customary approach. However, the results are given only in formal mathematical expressions. The present brief communication numerically evaluates the rigorous collisional damping rates by considering the case of plasma particles with Maxwellian velocity distribution function so as to assess the consequence of the rigorous formalism in a quantitative manner. Comparison with the heuristic ("Spitzer") formula shows that the accurate damping rates are much lower in magnitude than the conventional expression, which implies that the traditional approach over-estimates the importance of attenuation of plasma waves by collisional relaxation process. Such a finding may have a wide applicability ranging from laboratory to space and astrophysical plasmas. Published by AIP Publishing.

[http://dx.doi.org/10.1063/1.4953802]

In a recently published paper, ${ }^{1}$ the formalism of plasma kinetic theory was revisited, and a set of coupled equations were derived, which describe the dynamical evolution of the velocity distribution functions of plasma particles and the spectral wave energy densities associated with electrostatic waves. Reference 1 follows the standard weak turbulence perturbative ordering, except that unlike the textbook approaches, which take into account only the collective eigenmodes in the linear and nonlinear wave-particle interactions, the new formalism includes the effects of noncollective fluctuations emitted by thermal particles. It is shown that the non-collective fluctuations, which had been largely ignored in the literature hitherto, are responsible for collisional effects in both the particle and wave equations. Specifically, Ref. 1 demonstrates that the inclusion of noncollective part of thermal fluctuations leads to the collision integral, while the collective eigenmodes are responsible for the usual quasi-linear diffusion (plus the velocity friction) term(s) in the particle kinetic equation. As for the collectively excited waves, which satisfy the dispersion relations, and are thus eigenmodes, the influence of non-collective thermal fluctuations rigorously lead to the collisional wave damping of the collective waves, as well as the emission of these waves by particle collisions (i.e., bremsstrahlung emission of electrostatic eigenmodes). Such a derivation, without any ad hoc additions, was done for the first time.

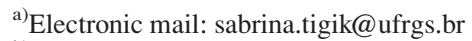

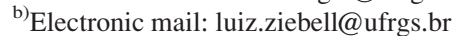

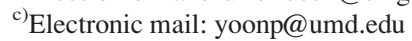

If one is interested only in the collisional relaxation for collision-dominated plasmas, then transport processes can be legitimately discussed solely on the basis of well-known collisional kinetic equation. ${ }^{2,3}$ Collisional transport is important for high density plasmas such as in the solar interior. In the opposite limit, if one's concern is only on relaxation processes that involve collective oscillations and waves, then various nonlinear theories of plasma turbulence may be employed. $^{4-7}$ Collective processes dominate rarefied plasmas, which characterize most of the heliosphere, interstellar, and intergalactic environments.

It is the dichotomy that separates the purely collisional versus purely collective descriptions that had not been rigorously bridged until the recent work. ${ }^{1}$ There are intermediate situations where both collisional and collective processes must be treated together, such as the solar $\mathrm{x}$ ray bremsstrahlung radiation sources, ${ }^{8-10}$ or in the Earth's ionospheric plasma where collisional conductivity becomes important. ${ }^{11}$ (Note that for the Earth's ionosphere, the dominant collisional process is the charged particle collisions with the neutrals, however.) For such situations, there was a general lack of satisfactory theories, which one may bring to bear in order to address the necessary physics, until recently. Instead, it had been a common practice to introduce collisional damping in the wave evolution as an indirect effect of assuming a collisional operator in the particle equation, and define an effective collision frequency. ${ }^{8-10,12-16}$ However, such a procedure is tantamount to inserting the collisional dissipation by hand, as it were, to the governing microscopic equation which describes fundamentally collision-free situation. 
Consequently, strictly speaking, the method is at best, heuristic. Nevertheless, such an ad hoc prescription is widely practiced in the plasma physics literature.

Thus, in the literature, often a governing equation is adopted

$$
\left[\frac{\partial}{\partial t}+\mathbf{v} \cdot \nabla+e_{a}\left(\mathbf{E}+\frac{\mathbf{v}}{c} \times \mathbf{B}\right) \cdot \frac{\partial}{\partial \mathbf{p}}\right] f_{a}=C_{a}\left(f_{a}\right),
$$

where $C_{a}\left(f_{a}\right)$ represents the collision integral and $a$ denotes particle species ( $a=e$ for electrons, $a=i$ for ions). If $f_{a}, \mathbf{E}$ and $\mathbf{B}$ in the above represent the averaged one-particle distribution function and average fields, then Eq. (1) represents the correct collisional kinetic equation. ${ }^{2,3}$ However, if these represent the total (average plus fluctuation), then they become microscopic one-particle distribution function and microscopic fields. For such a case, the right-hand side of Eq. (1) should be zero, since microscopic equations are reversible. As shown in Ref. 1, the irreversibility (signified by collision operator on the right-hand side) enters the problem only as a result of statistical averages and the loss of information. Nonetheless, the standard procedure in the literature is to interpret $f_{a}$ and field vectors as microscopic quantities, and employ expansion for small-amplitude perturbations. Upon replacing the collision operator by an effective collision frequency, $C_{a}\left(f_{a}\right) \approx-\nu_{\text {coll }} f_{a}$, the effective particle collision frequency is absorbed into the wave-particle resonance condition, and ends up as part of the imaginary part of the wave frequency, corresponding to a damping effect on the waves. As a consequence of the above-described recipe, known as the "Spitzer approximation" in the literature, one may obtain the collisional damping rate for Langmuir waves, given by

$$
\gamma_{\text {coll }}=-\frac{\pi n_{e} e^{4} \ln \Lambda}{m_{e}^{2} v_{T e}^{3}}
$$

where $v_{T e}=\left(2 T_{e} / m_{e}\right)^{1 / 2}$ is the electron thermal speed and $\Lambda=\lambda_{D e} T_{e} / e^{2}=4 \pi n_{e} \lambda_{D e}^{3}$ is a constant. Note that $\Lambda$ represents the total number of electrons in a sphere whose radius is equal to the Debye length, $\lambda_{D e}=\left[T_{e} /\left(4 \pi n_{e} e^{2}\right)\right]^{1 / 2}$. Here, $m_{e}$, $T_{e}$, and $n_{e}$ stand for electron mass, electron temperature (in the unit of energy), and electron density, respectively. Note that Eq. (2) implies that the collisional damping rate is constant and does not depend on wave vector (or wave frequency).

Reference 1 , in contrast, shows that the accurate collisional damping rates for plasma waves, that is, Langmuir $(L)$ and ion-acoustic $(S)$ waves, are much more complicated that is indicated by the approximate formula (2) in that the correct formulae exhibit dependence on wave number (and thus, frequency). However, the final results were given only in terms of formal expressions so that it is difficult to assess the consequence of the new formulation. The purpose of the present brief communication is to carry out numerical analysis so that one may understand the significance, or lack thereof, of the new findings in a quantitative way.

We start with the formal and rigorous expression for the collisional damping rates for $L$ and $S$ waves, as given by Eq. (4.44) in Ref. 1

$$
\begin{aligned}
\gamma_{\mathbf{k}}^{L(\text { coll })}= & \omega_{\mathbf{k}}^{L} \frac{4 n_{e} e^{4} \omega_{p e}^{2}}{T_{e}^{2}} \int d \mathbf{k}^{\prime} \frac{\left(\mathbf{k} \cdot \mathbf{k}^{\prime}\right)^{2} \lambda_{D e}^{4}}{k^{2} k^{\prime 4}\left|\epsilon\left(\mathbf{k}^{\prime}, \omega_{\mathbf{k}}^{L}\right)\right|^{2}} \\
& \times\left(1+\frac{T_{e}}{T_{i}}+\left(\mathbf{k}-\mathbf{k}^{\prime}\right)^{2} \lambda_{D e}^{2}\right)^{-2} \\
& \times \int d \mathbf{v} \mathbf{k}^{\prime} \cdot \frac{\partial F_{e}(\mathbf{v})}{\partial \mathbf{v}} \delta\left(\omega_{\mathbf{k}}^{L}-\mathbf{k}^{\prime} \cdot \mathbf{v}\right), \\
\gamma_{\mathbf{k}}^{S(\text { coll })}= & \mu_{\mathbf{k}} \omega_{\mathbf{k}}^{L} \frac{n_{e} e^{4} \omega_{p e}^{2}}{T_{e}^{2}} \int d \mathbf{k}^{\prime} \frac{1}{k^{2} k^{\prime 4}\left|\epsilon\left(\mathbf{k}^{\prime}, \omega_{\mathbf{k}}^{S}\right)\right|^{2}} \\
& \times\left(1+\frac{T_{e}}{T_{i}}+\left(\mathbf{k}-\mathbf{k}^{\prime}\right)^{2} \lambda_{D e}^{2}\right)^{-2} \\
& \times\left(1+\frac{2 T_{e} \mathbf{k} \cdot \mathbf{k}^{\prime}}{T_{i}} \frac{k^{2}}{k^{2}} \int \mathbf{v} \delta\left(\omega_{\mathbf{k}}^{S}-\mathbf{k}^{\prime} \cdot \mathbf{v}\right)\right. \\
& \times \mathbf{k}^{\prime} \cdot \frac{\partial}{\partial \mathbf{v}}\left(F_{e}(\mathbf{v})+\frac{m_{e}}{m_{i}} F_{i}(\mathbf{v})\right) .
\end{aligned}
$$

In the above equations, $\omega_{\mathbf{k}}^{L}=\omega_{p e}\left(1+\frac{3}{2} k^{2} \lambda_{D e}^{2}\right)$ and $\omega_{\mathbf{k}}^{S}$ $=\omega_{p e} k \lambda_{D e} \sqrt{\frac{m_{e}}{m_{i}} \frac{1+3 T_{i} / T_{e}}{1+k^{2} \lambda_{D e}^{2}}}$ designate Langmuir and ion-sound mode dispersion relations, respectively, $m_{i}$ and $T_{i}$ being the ion (proton) mass and temperature, respectively, and $\omega_{p e}=\left(4 \pi n_{e} e^{2} / m_{e}\right)^{1 / 2}$ is the plasma frequency. The ensemble-averaged one-particle distribution function $F_{a}(\mathbf{v})$ is normalized to unity, $\int d \mathbf{v} F_{a}(\mathbf{v})=1$. The quantity $\mu_{\mathbf{k}}$ is defined by $\mu_{\mathbf{k}}=k^{3} \lambda_{D e}^{3} \sqrt{\frac{m_{e}}{m_{i}}\left(1+\frac{3 T_{i}}{T_{e}}\right)}$, and $\epsilon\left(\mathbf{k}, \omega_{\mathbf{k}}^{L}\right)$ and $\epsilon\left(\mathbf{k}, \omega_{\mathbf{k}}^{S}\right)$ are the dielectric constants

$$
\epsilon(\mathbf{k}, \omega)=1+\sum_{a} \frac{\omega_{p a}^{2}}{k^{2}} \int d \mathbf{v} \frac{\mathbf{k} \cdot \partial F_{a} / \partial \mathbf{v}}{\omega-\mathbf{k} \cdot \mathbf{v}+i 0}
$$

Evidently, Eqs. (3) and (4) are far more sophisticated than the simple expression (2). The question is what is the actual implication of these results? Specifically, to what extent does the approximation (2) conform with the rigorous results (3) and (4), and if not, what is the extent of the discrepancy?

In order to quantitatively analyze Eqs. (3) and (4), it is advantageous to introduce suitable dimensionless quantities

$$
\mathbf{u}=\frac{\mathbf{v}}{v_{T e}}, \quad z=\frac{\omega}{\omega_{p e}}, \quad \mathbf{q}=\frac{\mathbf{k} v_{T e}}{\omega_{p e}}=\mathbf{k} \sqrt{2} \lambda_{D e}
$$

and rewrite the collisional damping rates (3) and (4) in normalized form

$$
\begin{aligned}
\gamma_{\mathbf{q}}^{L(\text { coll })} \equiv & \frac{\gamma_{\mathbf{k}}^{L(\text { coll })}}{\omega_{p e}}=\frac{2 g z_{\mathbf{q}}^{L}}{q^{2}} \int d \mathbf{q}^{\prime} \frac{\left(\mathbf{q} \cdot \mathbf{q}^{\prime}\right)^{2}}{q^{\prime 4}\left|\epsilon\left(\mathbf{q}^{\prime}, z_{\mathbf{q}}^{L}\right)\right|^{2}} \\
& \times\left(1+\frac{T_{e}}{T_{i}}+\frac{\left(\mathbf{q}-\mathbf{q}^{\prime}\right)^{2}}{2}\right)^{-2} \\
& \times \int d \mathbf{u} \mathbf{q}^{\prime} \cdot \frac{\partial \Phi_{e}(\mathbf{u})}{\partial \mathbf{u}} \delta\left(z_{\mathbf{q}}^{L}-\mathbf{q}^{\prime} \cdot \mathbf{u}\right),
\end{aligned}
$$




$$
\begin{aligned}
\gamma_{\mathbf{q}}^{S(\text { coll }) \equiv} \equiv & \frac{\gamma_{\mathbf{q}}^{S(\text { coll })}}{\omega_{p e}}=\frac{2 g z_{\mathbf{q}}^{L}}{q^{2}} \int \frac{d \mathbf{q}^{\prime}}{q^{\prime 4}\left|\epsilon\left(\mathbf{q}^{\prime}, z_{\mathbf{q}}^{S}\right)\right|^{2}} \\
& \times\left(1+\frac{T_{e}}{T_{i}}+\frac{\left(\mathbf{q}-\mathbf{q}^{\prime}\right)^{2}}{2}\right)^{-2} \\
& \times\left(1+\frac{2 T_{e}}{T_{i}} \frac{\mathbf{q} \cdot \mathbf{q}^{\prime}}{q^{2}}\right) \int d \mathbf{u} \delta\left(z_{\mathbf{q}}^{S}-\mathbf{q}^{\prime} \cdot \mathbf{u}\right) \\
& \times \mathbf{q}^{\prime} \cdot \frac{\partial}{\partial \mathbf{u}}\left(\Phi_{e}(\mathbf{u})+\frac{m_{e}}{m_{i}} \Phi_{i}(\mathbf{u})\right),
\end{aligned}
$$

where in dimensionless form, the dispersion relations are given by $z_{\mathbf{q}}^{L}=1+\frac{3 q^{2}}{4}$ and $z_{\mathbf{q}}^{S}=q \sqrt{\frac{m_{e}}{m_{i}} \frac{1+3 T_{i} / T_{e}}{2+q^{2}}}$. In Eqs. (6) and (7), the quantity $g$ is defined by

$$
g=\frac{1}{2^{3 / 2}(4 \pi)^{2} n_{e} \lambda_{D e}^{3}}=\frac{1}{2^{3 / 2}(4 \pi \Lambda)},
$$

which is related to the parameter $\Lambda$ discussed earlier. The quantity $g$ is an effective "plasma parameter" in that it is related to the inverse of the number of particles in a "Debye sphere."

Let us assume that ions and electrons have isotropic Maxwellian velocity distributions

$$
\Phi_{a}(\mathbf{u})=v_{e}^{3} F_{a}(\mathbf{v})=\frac{1}{\pi^{3 / 2}}\left(\frac{m_{a}}{m_{e}} \frac{T_{e}}{T_{a}}\right)^{3 / 2} \exp \left(-\frac{m_{a}}{m_{e}} \frac{T_{e}}{T_{a}} u^{2}\right) .
$$

Then the dielectric constants appearing in the denominators of Eqs. (3) and (4) are given by the following:

$$
\begin{aligned}
& \epsilon\left(\mathbf{q}^{\prime}, z_{\mathbf{q}}^{L}\right)=1+\frac{2}{q^{\prime 2}}\left[1+\frac{z_{\mathbf{q}}^{L}}{q^{\prime}} Z\left(\frac{z_{\mathbf{q}}^{L}}{q^{\prime}}\right)\right], \\
\epsilon\left(\mathbf{q}^{\prime}, z_{\mathbf{q}}^{S}\right)= & 1+\frac{2}{q^{\prime 2}}\left[1+\frac{z_{\mathbf{q}}^{S}}{q^{\prime}} Z\left(\frac{z_{\mathbf{q}}^{S}}{q^{\prime}}\right)\right] \\
+ & \frac{T_{e}}{T_{i}} \frac{2}{q^{\prime 2}}\left\{1+\left(\frac{m_{i}}{m_{e}} \frac{T_{e}}{T_{i}}\right)^{1 / 2} \frac{z_{\mathbf{q}}^{S}}{q^{\prime}} Z\left[\left(\frac{m_{i}}{m_{e}} \frac{T_{e}}{T_{i}}\right)^{1 / 2} \frac{z_{\mathbf{q}}^{S}}{q^{\prime}}\right]\right\} .
\end{aligned}
$$

For Maxwellian velocity distribution (9), the velocity integral $\int d \mathbf{u}$ in Eqs. (6) and (7) can be carried out analytically upon making use of the resonance delta function conditions. One may also perform the angular integration associated with the $\mathbf{q}^{\prime}$ vector integral, which reduces Eqs. (6) and (7) in the form that involves a single $q^{\prime}$ integration

$$
\begin{aligned}
\gamma_{\mathbf{q}}^{L(\text { coll })}= & -\left(16 \pi^{1 / 2} g\right) \frac{\left(z_{\mathbf{q}}^{L}\right)^{2}}{q^{2}} \int_{0}^{\infty} \frac{d q^{\prime}}{\left|\epsilon\left(q^{\prime}, z_{\mathbf{q}}^{L}\right)\right|^{2}} \\
& \times\left(\frac{2 B^{2}-A^{2}}{B^{2}-A^{2}}-\frac{B}{A} \ln \frac{B+A}{B-A}\right) \frac{1}{q^{\prime 3}} \exp \left(-\frac{\left(z_{\mathbf{q}}^{L}\right)^{2}}{q^{\prime 2}}\right),
\end{aligned}
$$

$$
\begin{aligned}
\gamma_{\mathbf{q}}^{S(\text { coll })}= & -\left(16 \pi^{1 / 2} g\right) \frac{\mu_{\mathbf{q}} z_{\mathbf{q}}^{L} z_{\mathbf{q}}^{S}}{q^{2}} \int_{0}^{\infty} \frac{d q^{\prime}}{\left|\epsilon\left(q^{\prime}, z_{\mathbf{q}}^{S}\right)\right|^{2}} \\
& \times\left[\frac{4}{B^{2}-A^{2}}-\frac{T_{e}}{T_{i}} \frac{q^{\prime}}{q} \frac{1}{q^{2} q^{\prime 2}}\left(\frac{2 A B}{B^{2}-A^{2}}-\ln \frac{B+A}{B-A}\right)\right] \\
& \times \sum_{a=e, i} \frac{T_{e}}{T_{a}}\left(\frac{m_{a}}{m_{e}} \frac{T_{e}}{T_{a}}\right)^{1 / 2} \frac{1}{q^{\prime 3}} \exp \left(-\frac{m_{a}}{m_{e}} \frac{T_{e}}{T_{a}} \frac{\left(z_{\mathbf{q}}^{S}\right)^{2}}{q^{\prime 2}}\right),
\end{aligned}
$$

where we have defined

$$
\begin{aligned}
& A=-2 q q^{\prime}, \\
& B=2\left(1+\frac{T_{e}}{T_{i}}\right)+q^{2}+q^{\prime 2} .
\end{aligned}
$$

For reference, the customary heuristic collisional damping rate (2), derived under the "Spitzer approximation," which is applicable for Langmuir wave, is given in normalized form by

$$
\bar{\gamma}_{\text {coll }} \equiv \frac{\gamma_{\text {coll }}}{\omega_{p e}}=-\frac{\pi n_{e} e^{4} \ln \Lambda}{m_{e}^{2} v_{T e}^{3} \omega_{p e}}=-\pi g \ln \left(\frac{1}{2^{3 / 2}(4 \pi g)}\right) .
$$

For comparison, we also discuss the collisionless damping, also known as Landau damping, which is well-known. From Eq. (3.24) of Ref. 1, we have the Landau damping rates for $L$ and $S$ waves

$$
\begin{aligned}
\gamma_{\mathbf{k}}^{L} & =\frac{\pi \omega_{\mathbf{k}}^{L} \omega_{p e}^{2}}{2 k^{2}} \int d \mathbf{v} \mathbf{k} \cdot \frac{\partial F_{e}(\mathbf{v})}{\partial \mathbf{v}} \delta\left(\omega_{\mathbf{k}}^{L}-\mathbf{k} \cdot \mathbf{v}\right) \\
\gamma_{\mathbf{k}}^{S} & =\frac{\pi \mu_{\mathbf{k}} \omega_{\mathbf{k}}^{L} \omega_{p e}^{2}}{2 k^{2}} \int d \mathbf{v} \mathbf{k} \cdot \frac{\partial}{\partial \mathbf{v}}\left(F_{e}(\mathbf{v})+\frac{m_{e}}{m_{i}} F_{i}(\mathbf{v})\right) \delta\left(\omega_{\mathbf{k}}^{S}-\mathbf{k} \cdot \mathbf{v}\right),
\end{aligned}
$$

which are textbook results. Making use of dimensionless variables, the above expressions are rewritten as

$$
\begin{aligned}
& \gamma_{\mathbf{q}}^{L}=-\frac{\pi^{1 / 2}\left(z_{\mathbf{q}}^{L}\right)^{2}}{q^{3}} \exp \left(-\frac{\left(z_{\mathbf{q}}^{L}\right)^{2}}{q^{2}}\right), \\
& \gamma_{\mathbf{q}}^{S}=-\frac{\pi^{1 / 2} \mu_{\mathbf{q}} z_{\mathbf{q}}^{L} z_{\mathbf{q}}^{S}}{q^{3}} \sum_{a=e, i} \frac{T_{e}}{T_{a}}\left(\frac{m_{a}}{m_{e}} \frac{T_{e}}{T_{a}}\right)^{1 / 2} \exp \left(-\frac{m_{a}}{m_{e}} \frac{T_{e}}{T_{a}} \frac{\left(z_{\mathbf{q}}^{S}\right)^{2}}{q^{2}}\right) .
\end{aligned}
$$

In Fig. 1, we plot the normalized collisional $L$ mode damping rate divided by $g, \gamma_{q}^{L(\text { coll })} / g$, as a function of dimensionless wave number $q$, for three values of the temperature ratio $T_{e} / T_{i}=10$ (red), 7 (black), and 4 (blue). It is shown that for the range of temperature ratios considered, the damping rate is maximum for $q$ between 5 and 9, approximately, and that the growth rate increases with decreasing temperature ratio $T_{e} / T_{i}$, for the entire range of wavelengths. In contrast, the approximate collisional damping rate divided by $g$, $\bar{\gamma}_{\text {coll }} / g=\pi \ln \left[2^{3 / 2}(4 \pi g)\right]$, is independent of the normalized wave number $q$, but the result depends on $g$. In general, the 


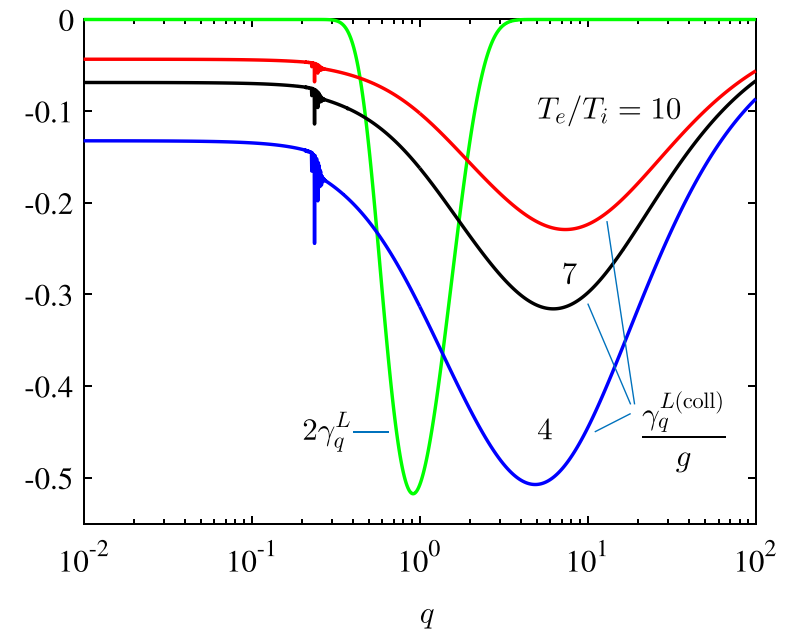

FIG. 1. Normalized collisional damping for $L$ waves, $\gamma_{q}^{L \text { (coll) }} / g$, vs normalized wavenumber $q$, for three values of the ratio $T_{e} / T_{i}$. The dimensionless Landau damping rate $\gamma_{q}^{L}$ is also plotted in green. Note that the Landau damping rate is not divided by the factor $g$. The factor 2, which multiplies $\gamma_{q}^{L}$ is for the sake of visual presentation.

plasma parameter $g$ must be small by definition, so we consider several different choices, $g=10^{-10}, 10^{-8}, 10^{-6}$, and $10^{-4}$. For these choices, we find that $\bar{\gamma}_{\text {coll }} / g \sim-61.12$, $-46.6524,-32.1849$, and -17.7173 , which are all far higher in absolute value than those depicted in Fig. 1. This shows that the use of incorrect collisional damping rate may greatly over-estimate the actual damping rate.

We also superpose in Fig. 1, the collisionless (Landau) damping rate for Langmuir wave [i.e., the first equation in (17)] vs $q$ (green). We multiplied the damping rate by factor 2 for visual reason. Note that the Landau damping rate is not divided by the plasma parameter $g$, so that the actual magnitude of the "collisionless" damping rate will greatly exceed that of the "collisional" damping rate by factor $1 / g \gg 1$. This shows that over the range of wave numbers over which the most important linear and nonlinear wave-particle interactions are expected to take place, the collisional damping of the Langmuir wave will be practically ignorable. However, it is interesting to note that for small wave number domain $(q \ll 1)$ for which the Landau damping rate becomes negligible, the collisional damping rate remains finite. In the collisionless plasmas, the undamped Langmuir waves in the long wavelength regime are supposed to lead to the so-called condensation phenomenon, where the wave energy accumulates without undergoing Landau damping. Over a long time period, the Zakharov strong turbulence effect is supposed to come into play in order to dissipate the accumulated wave energy. ${ }^{17}$ However, the present finding suggests that the collisional damping may contribute to the dissipation of the Langmuir wave energy in such a wavelength regime. We also note that for large $q$, the collisionless (Landau) damping rate eventually becomes exponentially weak. In contrast, the collisional damping rate may overcome the Landau damping rate, which makes sense, since for extremely short wavelength the binary collisions may lead to the damping of plasma waves.

Before we close and for the sake of completeness, we plot in Fig. 2 the normalized collisional damping for $S$ waves

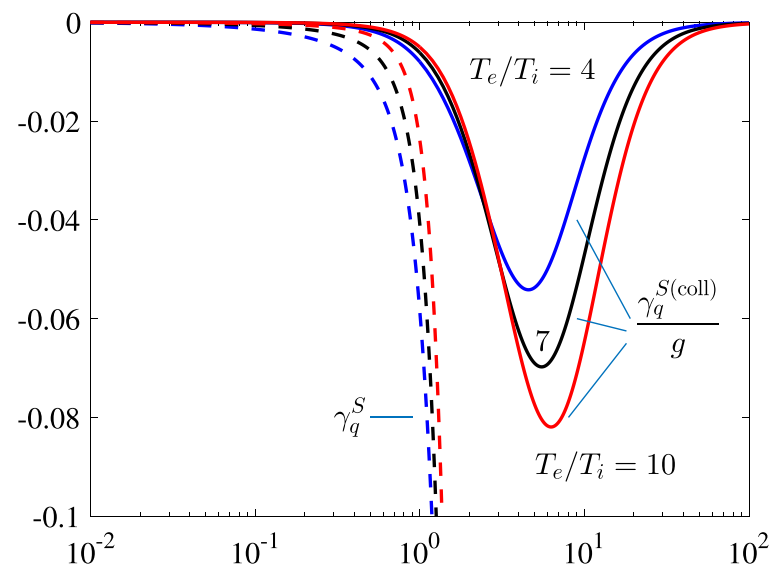

FIG. 2. Normalized collisional damping for $S$ waves, $\gamma_{q}^{S(\text { coll })} / g$, vs normalized wavenumber $q$, for three values of the ratio $T_{e} / T_{i}$.

divided by $g, \gamma_{q}^{S(\text { coll })} / g$, as a function of wave number $q$, for the same three values of the temperature ratio considered in Fig. 1, that is, $T_{e} / T_{i}=4,7$, and 10 . The same color scheme is used to indicate the three cases. Unlike the case of $L$ mode, the collisional damping rate for $S$ mode does not asymptotically approach a finite value for $q \rightarrow 0$. We also superpose the collisionless (Landau) damping rates for $S$ waves vs $q$, but since $\gamma_{q}^{S}$ depends on $T_{e} / T_{i}$, we use the same color scheme to indicate the three difference choices of $T_{e} / T_{i}$, except that we plot the collisionless damping rate with dashes. Again, we note that $\gamma_{q}^{S}$ is not divided by $g$, so that the actual damping rate is much higher in magnitude than the collisional damping rate $\gamma_{q}^{S(\text { coll })}$. In the case of $S$ mode, it becomes evident that the collisional damping plays no significant role whatsoever when compared against the collisionless damping, and thus the dynamical role of collisions on the dissipation of ion-sound mode damping becomes totally negligible.

In the present brief communication, we have investigated the formal collisional damping rates derived in Ref. 1, by numerical means. It is found that the collisional damping rates for Langmuir and ion-acoustic waves are much smaller than the conventional expressions, which means that the collisional damping has been over-estimated in the literature. While the collisional damping for ion-sound wave is totally negligible, the same for Langmuir wave becomes finite, albeit small, in the region of infinite wave length regime where collisionless Landau damping rate vanishes. Such a property may potentially provide the necessary dissipation mechanism in order to prevent the unchecked accumulation of wave energy for the long wavelength regime, known as the Langmuir condensation problem.

The importance of the present work is quite obvious. There are many physical situations where collisional and collective effects are both important, both in laboratory and space applications. The present analysis is based upon the recent work, ${ }^{1}$ which makes a simplifying assumption of electrostatic interaction in field-free plasmas. For more realistic applications, electromagnetic interaction in magnetized plasmas must be considered within the framework of the collisional weak turbulence theory. Reference 1 and the present work may represent the beginning of a new research paradigm. 
This work has been partially supported by CNPq (Brazil). P.H.Y. acknowledges support by NSF Grant Nos. AGS1147759 and AGS1550566 to the University of Maryland. The research at Kyung Hee University was supported by the BK21-Plus grant from the National Research Foundation (NRF), Korea. P.H.Y. also acknowledges the Science Award Grant from the GFT Foundation to the University of Maryland.

${ }^{1}$ P. H. Yoon, L. F. Ziebell, E. P. Kontar, and R. Schlickeiser, Phys. Rev. E 93, 033203 (2016).

${ }^{2}$ P. Helander and D. J. Sigmar, Collisional Transport in Magnetized Plasmas (Cambridge University Press, New York, 2002).

${ }^{3}$ G. P. Zank, Transport Processes in Space Physics and Astrophysics (Springer, New York, 2014).

${ }^{4}$ A. G. Sitenko, Fluctuations \& Non-linear Wave Interactions in Plasmas (Pergamon, New York, 1982).
${ }^{5}$ D. B. Melrose, Instabilities in Space and Laboratory Plasmas (Cambridge University Press, New York, 1986).

${ }^{6} \mathrm{R}$. Balescu, Aspects of Anomalous Transport in Plasmas (Institute of Physics Publishing, Philadelphia, 2005).

${ }^{7}$ R. A. Treumann and W. Baumjohann, Advanced Space Plasma Physics (Imperial College Press, London, 2001).

${ }^{8}$ K. G. McClements, Astron. Astrophys. 175, 255 (1987).

${ }^{9}$ I. G. Hannah, E. P. Kontar, and O. K. Sirenko, Astrophys. J. Lett. 707, L45 (2009)

${ }^{10}$ H. Ratcliffe and E. P. Kontar, Astron. Astrophys. 562, A57 (2014).

${ }^{11}$ M. C. Kelley, The Earth's Ionosphere (Academic Press, New York, 1989).

${ }^{12}$ G. G. Comisar, Phys. Fluids 6, 76 (1963).

${ }^{13}$ W. P. Wood and B. W. Ninham, Aust. J. Phys. 22, 453 (1969).

${ }^{14}$ D. B. Melrose, Plasma Astrophysics (Gordon and Breach, New York, 1980).

${ }^{15}$ A. O. Benz, Plasma Astrophysics (Kluwer, Dordrecht, 2002).

${ }^{16}$ E. M. Lifshitz and L. P. Pitaevskii, Physical Kinetics (Pergamon, New York, 1981).

${ }^{17}$ V. E. Zakharov, Sov. Phys. JETP 35, 908 (1972). 